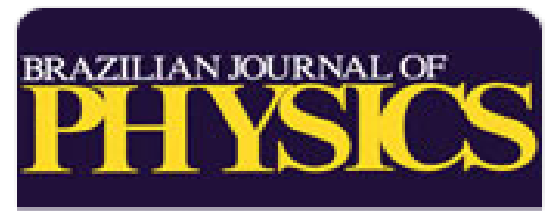

Brazilian Journal of Physics

ISSN: 0103-9733

luizno.bjp@gmail.com

Sociedade Brasileira de Física

Brasil

Bredice, F.; Borges, F. O.; Di Rocco, H. O.; Mercado, R. S.; Villagrán-Muniz, M.; Palleschi, V. A Procedure for Estimating the Electron Temperature and the Departure of the LTE Condition in a

Time-Dependent, Spatially Homogeneous, Optically Thin Plasma

Brazilian Journal of Physics, vol. 43, núm. 4, agosto, 2013, pp. 239-246

Sociedade Brasileira de Física

Sâo Paulo, Brasil

Available in: http://www.redalyc.org/articulo.oa?id=46427890005

How to cite

Complete issue

- More information about this article

Journal's homepage in redalyc.org

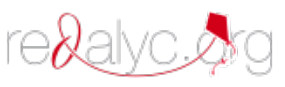

Scientific Information System 


\title{
A Procedure for Estimating the Electron Temperature and the Departure of the LTE Condition in a Time-Dependent, Spatially Homogeneous, Optically Thin Plasma
}

\author{
F. Bredice • F. O. Borges • H. O. Di Rocco • R. S. Mercado • \\ M. Villagrán-Muniz $\cdot$ V. Palleschi
}

Received: 31 May 2012 / Published online: 25 June 2013

(C) Sociedade Brasileira de Física 2013

\begin{abstract}
We present a method to estimate the temperature of transient plasmas and their degree of departure from local thermodynamic equilibrium conditions. Our method is based on application of the Saha-Boltzmann equations on the temporal variation of the intensity of the spectral lines of the plasma, under the assumption that the plasmas at the different times when the spectra were obtained are in local thermodynamic equilibrium. The method requires no knowledge of the spectral efficiency of the spectrometer/detector, transition probabilities of the considered lines, or degeneracies of the upper and lower levels. Provided that the conditions of optically thin, homogeneous plasma in local thermodynamic equilibrium are satisfied, the accuracy of the procedure is limited only by the precision
\end{abstract}

\section{F. Bredice}

Centro de Investigaciones Opticas, P.O. Box 124 C. P.1900,

La Plata, Argentina

F. O. Borges $(\square)$

Laboratório de Plasma e Espectroscopia Atômica, Instituto de Física, Universidade Federal Fluminense, 24210-340 Niterói,

Rio de Janeiro, Brazil

e-mail: borges@if.uff.br

H. O. Di Rocco

Instituto de Física Arroyo Seco (IFAS), Universidad Nacional del Centro, Pinto 399, Tandil, Argentina

\section{R. S. Mercado}

Grupo de Espectroscopia Óptica de Emisión y Láser (GEOEL),

Universidad del Atlántico, A.A. 1890, Barranquilla, Colombia

M. Villagrán-Muniz

Laboratorio de Fotofísica, Centro de Ciencias Aplicadas y

Desarrollo Tecnológico, Universidad Nacional Autónoma de

México, Apartado Postal 70-186, México 04510, Mexico

V. Palleschi

Applied Laser Spectroscopy Laboratory, ICCOM-CNR, Area della

Ricerca di Pisa, Via G. Moruzzi 1, 56124 Pisa, Italy with which the line intensities and densities can be determined at two different temperatures. The procedure generates an equation describing the temporal evolution of the electron number density of transient plasmas under local thermodynamic equilibrium conditions. The method is applied to the analysis of two laser-induced breakdown spectra of cadmium at different temperatures.

Keywords Laser plasma $\cdot$ Electron temperature $\cdot$ Electron number density $\cdot$ LTE $\cdot$ LIBS

\section{Introduction}

The electron plasma temperature, along with the electronnumber density and the densities of the different species in the plasma, is one of the main parameters characterizing the plasma [1-3]. The temperature must be known before the plasma can be analyzed by calibration-free laser-induced breakdown spectroscopy (LIBS) [4-6]. In conditions close to local thermodynamic equilibrium (LTE) [7], the Boltzmann plot method [8-15] usually determines the plasma temperature. Alternatively, when information about the electron number density is available and the intensities of emission lines from different ionization stages of the same element are measurable, the Saha-Boltzmann plot can be used [16], even though the application of the latter method to nonhomogeneous plasmas has been questioned [17]. However, even under ideal conditions, i. e., homogeneous plasmas under LTE conditions, the Boltzmann plot and the Saha-Boltzmann plot require previous knowledge of the spectral parameters of the reference lines, which are subject to large uncertainties, larger than $50 \%$ - especially in the case of the transition probabilities $A_{\mathrm{ki}}[18]$. In certain cases, the spectral parameters are not known at all. Moreover, the relative efficiency of the spectral equipment 
and of the collection optics must be known, at the wavelengths of the reference lines.

The alternative proposed here requires knowledge of no other line spectral parameters than the upper level energy $E_{\mathrm{k}}$ and is independent of the spectrometer efficiency. Comparison between the intensities of two emission lines of the same element two or more times after the laser breakdown is sufficient to estimate the plasma temperature.

\section{Mathematical Basis for the New Method}

Our approach is based on the variation of the spectral line intensities in time-dependent plasmas and follows a procedure we have recently proposed [19]. Assuming the usual conditions, of a homogeneous plasma in LTE [7] at the measurement times, a rather simple expression can be obtained to determine the plasma temperature.

In an optically thin plasma under LTE conditions, the spectrally integrated intensity of an emitted line is given by the expression

$I_{\mathrm{ki}}=\frac{h c}{4 \pi \lambda_{\mathrm{ki}}} \frac{A_{\mathrm{ki}} g_{\mathrm{k}} N \exp \left(-\frac{E_{\mathrm{k}}}{k_{B} T}\right)}{U(T)}$

where $I_{\mathrm{ki}}$ is the intensity of the transition from the state $k$ to a lower state $i, h$ is Planck's constant, $c$ is the speed of light, $\lambda_{\mathrm{ki}}$ is the transition-line wavelength, $A_{\mathrm{ki}}$ is the transition probability, $E_{\mathrm{k}}$ and $g_{\mathrm{k}}$ are the energy and the statistical weight of the level $k, N$ is the total density of emitting atoms, $U(T)$ is the partition function for the atomic species at temperature $T$, and $k_{\mathrm{B}}$ is the Boltzmann constant $[1,12]$.

Equation (1) can be rewritten in the form

$\ln \left(\frac{I_{\mathrm{ki}} \lambda_{\mathrm{ki}}}{g_{\mathrm{k}} A_{\mathrm{ki}}}\right)=-\frac{E_{\mathrm{k}}}{k_{\mathrm{B}} T}+\ln \left(\frac{h c N}{4 \pi U(T)}\right)$,

which constitutes the basis of the Boltzmann plot method, in which the left-hand side is plotted as a function of the upperlevel energy $\mathrm{E}_{\mathrm{k}}$. In the LTE approximation and in the absence of self-absorption $[2,12,19]$, points corresponding to the emission lines of the same species are expected to form a straight line with slope $-\frac{1}{k_{\mathrm{B}} T}$.

When Eq. (2) is written for the same line at two different temperatures, the difference between the two expression has the form

$\ln \left(\frac{I\left(T_{\mathrm{a}}\right)}{I\left(T_{\mathrm{b}}\right)}\right)=-\frac{E_{\mathrm{k}}}{k_{\mathrm{B}}}\left(\frac{1}{T_{\mathrm{a}}}-\frac{1}{T_{\mathrm{b}}}\right)+\ln \left(\frac{N\left(T_{\mathrm{a}}\right) U\left(T_{\mathrm{b}}\right)}{U\left(T_{\mathrm{a}}\right) N\left(T_{\mathrm{b}}\right)}\right)$

The ratio in the argument on the left-hand side of Eq. (3) is calculated for two lines $I_{1}$ and $I_{2}$ of the same species, and its logarithm is plotted as a function of $E_{\mathrm{k}}$. Again in the approximation of LTE and absence of self-absorption, the points corresponding to different emission lines of the same species are expected to align along a straight line, as in Fig. 1. From Eq. (3), we can see that the slope $m$ of this plot is given by the equality

$$
\begin{aligned}
m & =\frac{\ln \left(\frac{I_{2}\left(T_{\mathrm{a}}\right)}{I_{2}\left(T_{\mathrm{b}}\right)}\right)-\ln \left(\frac{I_{1}\left(T_{\mathrm{a}}\right)}{I_{1}\left(T_{\mathrm{b}}\right)}\right)}{E_{\mathrm{k} 2}-E_{\mathrm{k} 1}} \\
& =\frac{E_{\mathrm{k} 2}\left(\frac{1}{T_{\mathrm{b}}}-\frac{1}{T_{\mathrm{a}}}\right)-E_{\mathrm{k} 1}\left(\frac{1}{T_{\mathrm{b}}}-\frac{1}{T_{\mathrm{a}}}\right)}{k_{\mathrm{B}}\left(E_{\mathrm{k} 2}-E_{\mathrm{k} 1}\right)}=\frac{1}{k_{\mathrm{B}}}\left(\frac{1}{T_{\mathrm{b}}}-\frac{1}{T_{\mathrm{a}}}\right)
\end{aligned}
$$

Moreover, the Saha equation [20], written in terms of the intensities of spectral lines corresponding to different ionization stages of the same element, can be expressed in the form

$\frac{I_{\mathrm{ki}}}{I_{\mathrm{j} 1}}=\frac{A_{\mathrm{ki}} \lambda_{\mathrm{ki}}}{A_{\mathrm{j} 1} \lambda_{\mathrm{j} 1}} \frac{2 g_{\mathrm{k}}}{g_{\mathrm{j}}} \frac{\left(2 \pi m_{\mathrm{e}} k_{\mathrm{B}} T\right)^{3 / 2}}{h^{3}} \frac{1}{n_{\mathrm{e}}(T)} e^{-\frac{\left(\chi+E_{\mathrm{k}}-E_{\mathrm{j}}\right)}{k_{\mathrm{B}} T}}$

where $n_{\mathrm{e}}(T)$ is the (electron number) density at the temperature $T, m_{\mathrm{e}}$ is the electron mass, and $\chi(\mathrm{eV})$ is the ionization energy of the species. For practical purposes, it is convenient to rewrite Eq. (5) as the ratio of the intensities of the same lines at two different temperatures, which yields the relation

$\frac{I_{\mathrm{ki}}\left(T_{\mathrm{a}}\right)}{I_{\mathrm{jl}}\left(T_{\mathrm{a}}\right)} \frac{I_{\mathrm{jl}}\left(T_{\mathrm{b}}\right)}{I_{\mathrm{ki}}\left(T_{\mathrm{b}}\right)}=\left(\frac{T_{\mathrm{a}}}{T_{\mathrm{b}}}\right)^{3 / 2} \frac{n_{\mathrm{e}}\left(T_{\mathrm{b}}\right)}{n_{\mathrm{e}}\left(T_{\mathrm{a}}\right)} \exp \left[-\left(\chi+E_{\mathrm{k}}-E_{\mathrm{j}}\right) \frac{1}{k_{\mathrm{B}}}\left(\frac{1}{T_{\mathrm{a}}}-\frac{1}{T_{\mathrm{b}}}\right)\right]$

From Eq. (4) we know that

$\left(\frac{T_{\mathrm{a}}}{T_{\mathrm{b}}}\right)=k_{\mathrm{B}} T_{\mathrm{a}} m+1$,

a result that can be substituted in Eq. (6) to yield the equality

$k_{\mathrm{B}} T_{\mathrm{a}}=\left[\left(\frac{n_{\mathrm{e}}\left(T_{\mathrm{a}}\right)}{n_{\mathrm{e}}\left(T_{\mathrm{b}}\right)} \frac{I_{\mathrm{ki}}\left(T_{\mathrm{a}}\right)}{I_{\mathrm{ki}}\left(T_{\mathrm{b}}\right)} \frac{I_{\mathrm{jl}}\left(T_{\mathrm{b}}\right)}{I_{\mathrm{jl}}\left(T_{\mathrm{a}}\right)}\right)^{2 / 3} \exp \left[-\frac{2}{3}\left(\chi+E_{\mathrm{k}}-E_{\mathrm{j}}\right) m\right]-1\right] \frac{1}{m}$

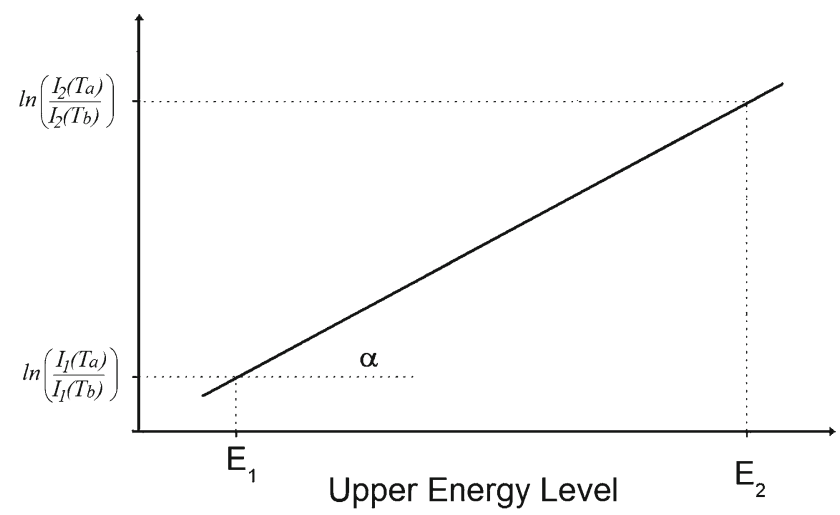

Fig. 1 Schematic plot of Eq. (3) 
The ratio $\frac{n_{\mathrm{e}}\left(T_{\mathrm{a}}\right)}{n_{\mathrm{e}}\left(T_{\mathrm{b}}\right)}$ on the right-hand side can be measured experimentally - via one of the methods in Ref. [8], for instance. Equation (8) therefore determines the plasma temperature $T_{\mathrm{a}}$, after which Eq. (4) determines $T_{\mathrm{b}}$. Over the traditional Boltzmann or Saha-Boltzmann plot methods, Eq. (8) has a definite advantage: it determines the electron temperature(s) independently of the spectral parameters $A_{\mathrm{ki}}$ of the studied transitions and of the equipment calibration, since the calibration constant cancels out of the quotient between the intensities of the same line at different temperatures. This advantage has a price, for we have to measure the line intensities at two distinct temperatures; moreover, as we shall see, at least three lines of different ionization stages should be used, while the application of the Boltzmann or Saha-Boltzmann plot method in principle calls for only two lines from a single element.

If information is available on more than the minimum necessary number of lines, Eq. (8) can be generalized. According to Eq. (4), the slope $m$ of the plot is the same for both neutral and ionized species of the same element. As indicated in Fig. 2, the linear regression of the two plots gives both $\mathrm{m}$ and the two intercepts $q_{1}$ and $q_{2}$, which can be interpreted as the values of the logarithms of $\frac{I_{\mathrm{ki}}\left(T_{\mathrm{a}}\right)}{I_{\mathrm{ki}}\left(T_{\mathrm{b}}\right)}$ and $\frac{I_{\mathrm{j}}\left(T_{\mathrm{a}}\right)}{I_{\mathrm{j} 1}\left(T_{\mathrm{b}}\right)}$ at $E_{\mathrm{k}}=0$ and $E_{\mathrm{j}}=0$. Substitution in Eq. (8) then yields the expression

$k_{\mathrm{B}} T_{\mathrm{a}}=\frac{\left[\left(\left(\frac{n_{\mathrm{c}}\left(T_{\mathrm{T}}\right)}{n_{\mathrm{e}}\left(T_{\mathrm{b}}\right)}\right)^{2 / 3} \exp \left[\frac{2}{3}\left(q_{1}-q_{2}-\chi_{m}\right)\right]\right)-1\right]}{m}$

In view of Eq. (9), we can evaluate the plasmatemperature relative error resulting from the experimental deviation of the measured electron number densities and fitting parameters $m, q_{1}$, and $q_{2}$, from the equality

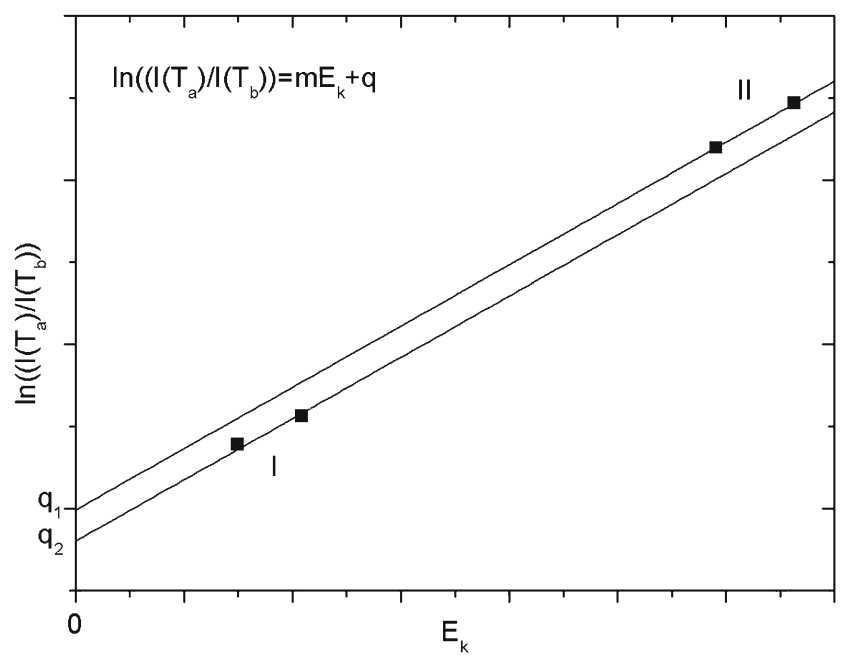

Fig. 2 The plot in Fig. 1 for neutral and ionized lines

$$
\begin{aligned}
& \left|\frac{\Delta T_{\mathrm{a}}}{T_{\mathrm{a}}}\right|=\frac{2}{3}\left|\left[\frac{\left(\frac{n_{\mathrm{e}}\left(T_{\mathrm{a}}\right)}{n_{\mathrm{e}}\left(T_{\mathrm{b}}\right)}\right)^{2 / 3} \exp \left[\frac{2}{3}\left(q_{1}-q_{2}-\chi_{m}\right)\right]}{\left(\frac{n_{\mathrm{e}}\left(T_{\mathrm{a}}\right)}{n_{\mathrm{e}}\left(T_{\mathrm{b}}\right)}\right)^{2 / 3} \exp \left[\frac{2}{3}\left(q_{1}-q_{2}-\chi_{m}\right)\right]-1}\right] \frac{\Delta\left(\frac{n_{\mathrm{e}}\left(T_{\mathrm{a}}\right)}{n_{\mathrm{e}}\left(T_{\mathrm{b}}\right)}\right)}{\frac{n_{\mathrm{e}}\left(T_{\mathrm{a}}\right)}{n_{\mathrm{e}}\left(T_{\mathrm{b}}\right)}}\right|+ \\
& +\frac{2}{3}\left|q_{1}\left[\frac{\left(\frac{n_{\mathrm{e}}\left(T_{\mathrm{a}}\right)}{n_{\mathrm{e}}\left(T_{\mathrm{b}}\right)}\right)^{2 / 3} \exp \left[\frac{2}{3}\left(q_{1}-q_{2}-\chi_{m}\right)\right]}{\left(\frac{n_{\mathrm{e}}\left(T_{\mathrm{a}}\right)}{n_{\mathrm{e}}\left(T_{\mathrm{b}}\right)}\right)^{2 / 3} \exp \left[\frac{2}{3}\left(q_{1}-q_{2}-\chi_{m}\right)\right]-1}\right] \frac{\Delta\left(q_{1}\right)}{q_{1}}\right|+ \\
& +\frac{2}{3}\left|q_{2}\left[\frac{\left(\frac{n_{\mathrm{e}}\left(T_{\mathrm{a}}\right)}{n_{\mathrm{e}}\left(T_{\mathrm{b}}\right)}\right)^{2 / 3} \exp \left[\frac{2}{3}\left(q_{1}-q_{2}-\chi_{m}\right)\right]}{\left(\frac{n_{\mathrm{e}}\left(T_{\mathrm{a}}\right)}{n_{\mathrm{e}}\left(T_{\mathrm{b}}\right)}\right)^{2 / 3} \exp \left[\frac{2}{3}\left(q_{1}-q_{2}-\chi_{m}\right)\right]-1}\right] \frac{\Delta\left(q_{2}\right)}{q_{2}}\right|+ \\
& +\left|\left(\frac{2}{3} \chi m\left[\frac{\left(\frac{n_{\mathrm{e}}\left(T_{\mathrm{a}}\right)}{n_{\mathrm{e}}\left(T_{\mathrm{b}}\right)}\right)^{2 / 3} \exp \left[\frac{2}{3}\left(q_{1}-q_{2}-\chi_{m}\right)\right]}{\left(\frac{n_{\mathrm{e}}\left(T_{\mathrm{a}}\right)}{n_{\mathrm{e}}\left(T_{\mathrm{b}}\right)}\right)^{2 / 3} \exp \left[\frac{2}{3}\left(q_{1}-q_{2}-\chi_{m}\right)\right]-1}\right]+1\right) \frac{\Delta(m)}{m}\right|+
\end{aligned}
$$

For plasmas such as most LIBS plasmas, showing timedependent line intensities approximately described by a decreasing exponential $I=I_{0} \exp \left[-\lambda\left(t-t_{0}\right)\right]$, from Eq. (8) we have that

$n_{\mathrm{e}}(t)=\frac{n_{\mathrm{e}}\left(t_{0}\right) \exp \left(-K\left(t-t_{0}\right)\right)}{\left(B\left(t-t_{0}\right)+1\right)^{3 / 2}}$

where $n_{\mathrm{e}}(t)$ and $n_{\mathrm{e}}\left(t_{0}\right)$ are the electron number densities at times $\mathrm{t}$ and $\mathrm{t}_{0}$ respectively, and $K$ and $B$ are numerical constants.

If the electron number density deviates from this behavior, the Saha-Boltzmann equation is not satisfied and the plasma is not in LTE. According to Ref. [7], the variation rate of the temperature and electron number density for homogeneous, transient plasmas must exceed the plasma relaxation time. Equation (11), along with the aforementioned considerations, provides a complementary check on the LTE conditions in LIBS experiments.

\section{Considerations Concerning the Application of the Procedure}

To test the new method, we used the computer software detailed in Ref. [21], which generates synthetic LIBS spectra

Table 1 Spectral parameters of the lines considered [18]

\begin{tabular}{lllllll}
\hline$\lambda(\mathcal{A})$ & Species & $g_{\mathrm{k}}$ & $g_{\mathrm{i}}$ & $A_{\mathrm{ki}}\left(\mathrm{s}^{-1}\right)$ & $E_{\mathrm{k}}\left(\mathrm{cm}^{-1}\right)$ & $E_{\mathrm{i}}\left(\mathrm{cm}^{-1}\right)$ \\
\hline $2,816.19$ & Al II & 1 & 3 & $3.83 \times 10^{8}$ & $9.5 \times 10^{4}$ & $5.99 \times 10^{4}$ \\
$3,082.16$ & Al I & 4 & 2 & $6.3 \times 10^{7}$ & $3.24 \times 10^{4}$ & 0 \\
$3,092.72$ & Al I & 6 & 4 & $7.4 \times 10^{7}$ & $3.24 \times 10^{4}$ & $1.12 \times 10^{2}$ \\
$3,944.02$ & Al I & 2 & 2 & $4.93 \times 10^{7}$ & $2.53 \times 10^{4}$ & 0 \\
$3,961.48$ & Al I & 2 & 4 & $9.8 \times 10^{7}$ & $2.53 \times 10^{4}$ & $1.12 \times 10^{2}$ \\
$4,663.07$ & Al II & 3 & 5 & $3.83 \times 10^{8}$ & $1.07 \times 10^{5}$ & $8.55 \times 10^{4}$ \\
\hline
\end{tabular}




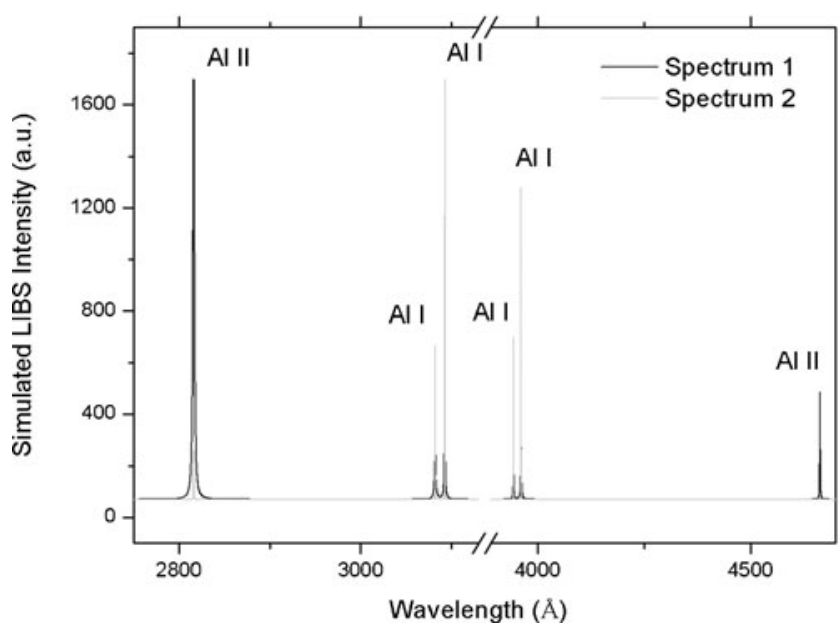

Fig. 3 Synthetic LIBS spectrum. Black curve, $T_{\mathrm{a}}=1.6 \mathrm{eV}$; $n_{\mathrm{e}}\left(T_{\mathrm{a}}\right)=4.0 \times 10^{17} \mathrm{~cm}^{-3} ;$ gray curve, $T_{\mathrm{b}}=1 \mathrm{eV} ; n_{\mathrm{e}}\left(T_{\mathrm{b}}\right)=0.3 \times 10^{17} \mathrm{~cm}^{-3}$

while accounting for self-absorption and inhomogeneity effects in the plasma. The synthetic spectra and the control over physical effects always present in real spectra give insight into the potential benefits resulting from application of the present method. As an example, we will consider an idealized spectrum constituted only by four Al I emission lines at 3,082.16, 3,092.72, 3,944.02, and 3,961.48 $\AA$, and two Al II line at 2,816.19 $\AA$ and 4,663.07 $\AA$, respectively. For the moment, we neglect self-absorption effects and consider a homogeneous plasma. We will also assume that the spectral coefficients of the two lines would correspond to the ones in Table 1 and in particular that the $A_{\mathrm{ki}}$ coefficients are known with negligible error.

Consider the two different synthetic spectra in Fig. 3, corresponding to the following conditions:

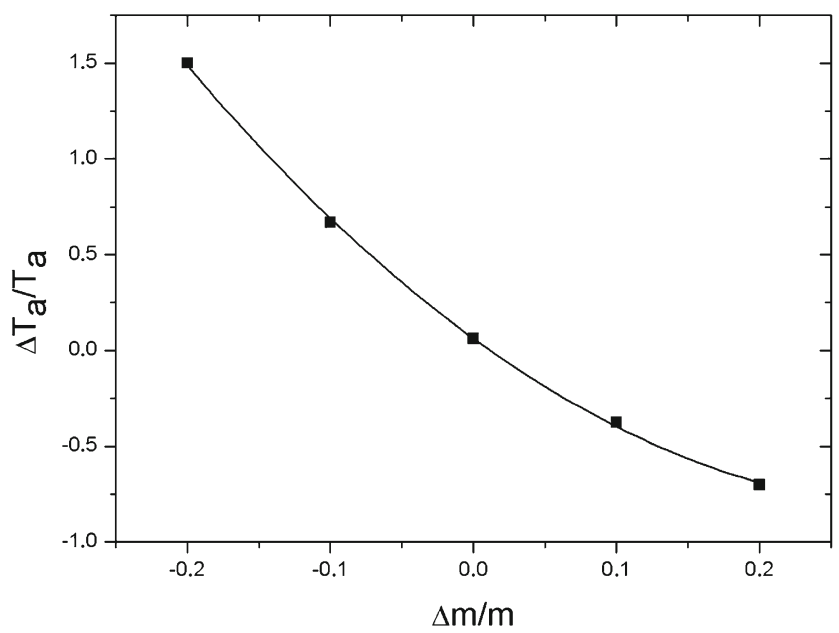

Fig. 4 Relative error in temperature determination as a function of the relative error on $m$. The continuous lines are guides to the eye obtained by fitting the numerical data with a second-order polynomial
Spectrum 1: $T_{\mathrm{a}}=1.6 \mathrm{eV} ; n_{\mathrm{e}}\left(T_{\mathrm{a}}\right)=4.0 \times 10^{17} \mathrm{~cm}^{-3}$

Spectrum 2: $T_{\mathrm{b}}=1 \mathrm{eV} ; n_{\mathrm{e}}\left(T_{\mathrm{b}}\right)=0.3 \times 10^{17} \mathrm{~cm}^{-3}$

In the ideal situation in Fig. 3, the fitting of the lines intensities yields $m=0.373, q_{1}=0.397$ and $q_{2}=0.016$. From these results, Eq. (9) gives $T_{\mathrm{a}}=1.70 \mathrm{eV}$. From Eq. (7), it also follows that $T_{\mathrm{b}}=1.04 \mathrm{eV}$. Even in this highly idealized situation, therefore, the small errors in the fitting of the synthetic lines introduce temperature deviations of the order of $5 \%$. The temperatures $T_{a}$ and $T_{b}$ were obtained without any information on the $A_{\mathrm{ki}}$ coefficients of the two lines.

In this situation, the error in the fitting parameter $m$ affects most strongly the computed $T_{\mathrm{a}}$. Figure 4 shows the relative error in the plasma temperature as a function of the relative error in the fitting parameter $\mathrm{m}$. An error of $10 \%$ on $m$ results in a plasma-temperature error of $60 \%$.

We can also compare the electron temperatures resulting from Eq. (9) and from the Saha-Boltzmann method for the same spectral lines. Since both approaches depend on the plasma electron-number density, it is instructive to plot the relative plasma-temperature deviations as functions of the relative density error (Fig. 5). The Saha-Boltzmann plot method gives reasonable temperatures, within $10 \%$ deviation from the exact ones, even for density uncertainties of $25 \%$. By contrast, under the same conditions, our method introduces $40 \%$ error.

\section{Experimental Application}

To test our method under experimental conditions, we have studied several $\mathrm{Cd}$ lines in laser-produced plasma spectra emitted from a pure-cadmium sample. Two experiments

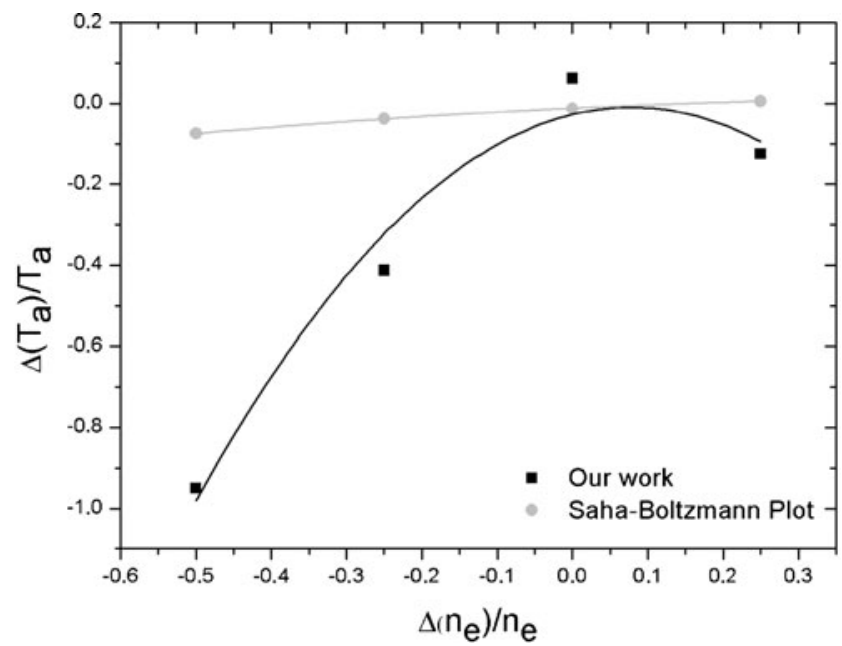

Fig. 5 Relative error in estimated temperature as a function of the relative error on the electron number density. The continuous lines are guides to the eye obtained by fitting the numerical data with a secondorder polynomial 
Table 2 Spectral parameters of the $\mathrm{Cd}$ lines considered in the experimental application

\begin{tabular}{llllllll}
\hline$\lambda(\mathcal{A})$ & Species & $g_{\mathrm{k}}$ & $g_{\mathrm{i}}$ & $A_{\mathrm{ki}}\left(\mathrm{s}^{-1}\right)$ & $E_{\mathrm{k}}\left(\mathrm{cm}^{-1}\right)$ & $E_{\mathrm{i}}\left(\mathrm{cm}^{-1}\right)$ & References \\
\hline $2,312.77$ & Cd II & 6 & 4 & $7.07 \times 10^{7}$ & $89,843.780$ & $46,618.550$ & {$[22]$} \\
$2,572.82$ & Cd II & 2 & 2 & $3.22 \times 10^{8}$ & $82,990.660$ & $44,136.080$ & {$[22,25]$} \\
$2,836.90$ & Cd I & 3 & 1 & $2.8 \times 10^{7}$ & $65,353.372$ & $30,113.990$ & {$[23,25]$} \\
$2,880.76$ & Cd I & 5 & 3 & $4.2 \times 10^{7}$ & $65,358.881$ & $30,656.087$ & {$[23,25]$} \\
$2,980.62$ & Cd I & 7 & 5 & $5.9 \times 10^{7}$ & $65,367.227$ & $31,826.952$ & {$[23,25]$} \\
$3,133.16$ & Cd I & 3 & 3 & $1.16 \times 10^{7}$ & $62,563.435$ & $30,656.087$ & {$[24]$} \\
$3,403.65$ & Cd I & 3 & 1 & $7.7 \times 10^{7}$ & $59,485.768$ & $30,113.990$ & {$[22,25]$} \\
\hline
\end{tabular}

were carried out with different time delay, 2 and $6 \mu \mathrm{s}$. The plasmas were generated in air at atmospheric pressure using a Q-switched Nd:YAG laser (Quantel, model Brilliant B, $1,064 \mathrm{~nm}$ wavelength, $6 \mathrm{~ns}$ pulse width, and $30 \mathrm{~mJ}$ energy). The laser was focused on a solid cadmium target by a biconvex lens of $75 \mathrm{~mm}$ focal length, forming the plasma over the target surface. The plasma light emission was collected on the spectrometer slit with a quartz cylindrical lens as guide. A Czerny-Turner monochromator-iCCD system (Andor Shamrock 500i-DH734-18F) recorded the emission spectrum. The Czerny-Turner, with $0.5 \mathrm{~m}$ focal length, 1,200 lines/nm grating blazed at $500 \mathrm{~nm}, 40 \mathrm{~nm}$ bandpass, and $50 \mu \mathrm{m}$ slit aperture, has a wavelength resolution of $0.06 \mathrm{~nm}$. The spectra were acquired with $50 \mathrm{~ns}$ gate time and 100 accumulation.

We studied the cadmium spectrum in detail from 200 to $700 \mathrm{~nm}$ in order to select the spectral lines that would be used in the test. We looked for nonresonant lines without spectral interferences, with known atomic parameters - degeneracy, level energy, and transition probability - and close to each other in the spectrum. Table 2 lists the selected lines and their parameters.

Our study covered two spectral regions. The spectrum in the first (second) region, which comprises Cd I (Cd II)

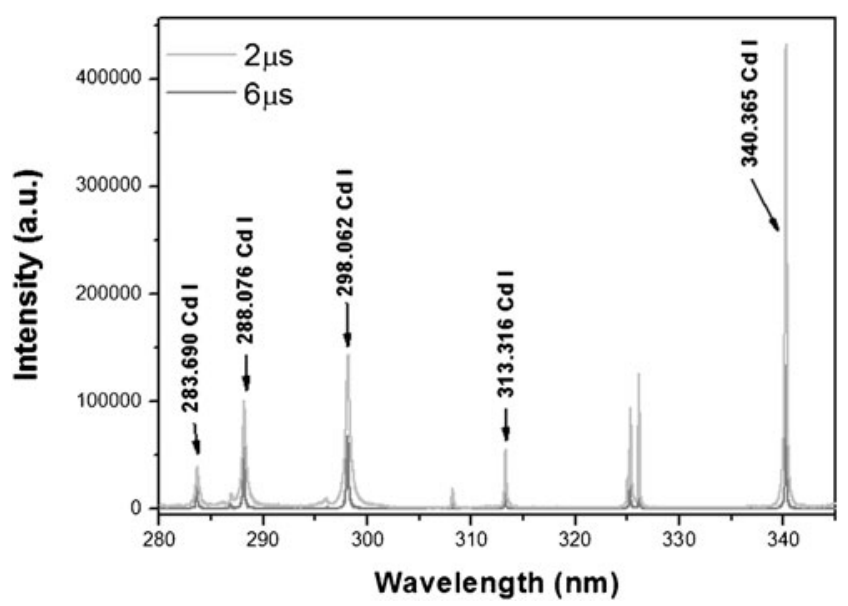

Fig. 6 Spectral region with Cd I lines emission lines and extends from 280 to $345 \mathrm{~nm}$ (230 to $260 \mathrm{~nm}$ ), is shown in Figs. 6 and 7. The atomic and ionic cadmium lines have been indicated in each figure.

In the spectrum measured with $2 \mu$ s delay, the electron density was calculated from optically thin $\mathrm{H} \alpha$-line, following the prescription in Ref. [26]. To measure the linewidths we resorted to Voigt fitting of the profile: the Gaussian contribution of the instrumental broadening was then subtracted to leave only the Lorentzian contribution associated with the Stark effect $\left(\Delta \lambda_{\text {Stark }}=0.949 \mathrm{~nm}\right)$, shown in Fig. 8. The resulting electron density is $n_{\mathrm{e}}(2 \mu \mathrm{s})=8.20 \times 10^{16} \mathrm{~cm}^{-3}$.

A alternative estimate of the electron density is given by Saha's equation. The resulting electron density then depends on the calibration of the detection system. With the cadmium calibrated spectrum, we use the procedure in Ref. [27] to calculate the electron density. Since the method uses atomic and ionic lines, we were able to obtain 10 electron densities for each spectrum, the average of which was subsequently calculated. The spectra with 2 and $6 \mu$ s delays yielded average densities of $<n_{\mathrm{e}}(2 \mu \mathrm{s})>=8.74 \times 10^{16} \mathrm{~cm}^{-3}$ and $<n_{\mathrm{e}}(6 \mu \mathrm{s})>=3.92 \times 10^{16} \mathrm{~cm}^{-3}$, respectively.

We have followed the procedure in Ref. [28] to obtain the Saha-Boltzmann plots in Fig. 9. For each time delay, the displayed linear regression yields good correlation. The

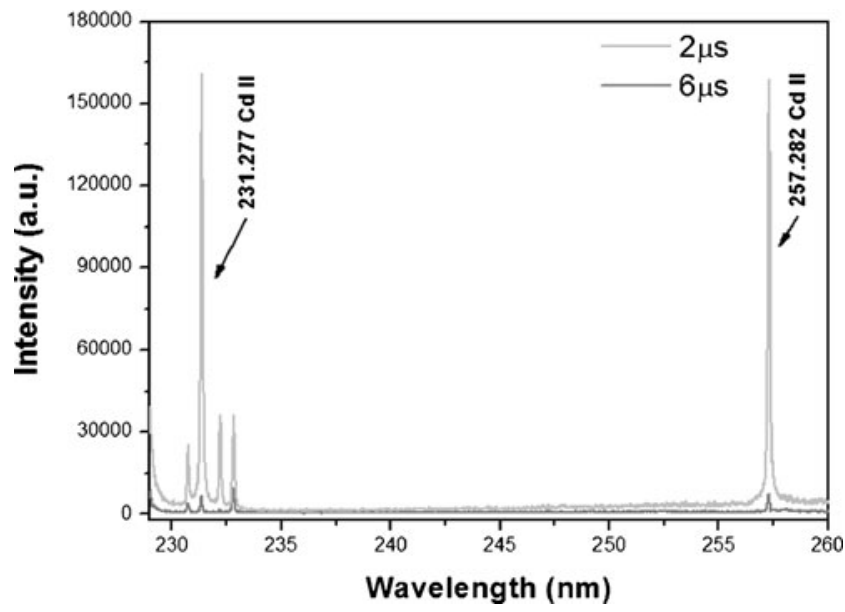

Fig. 7 Spectral region with Cd II lines 


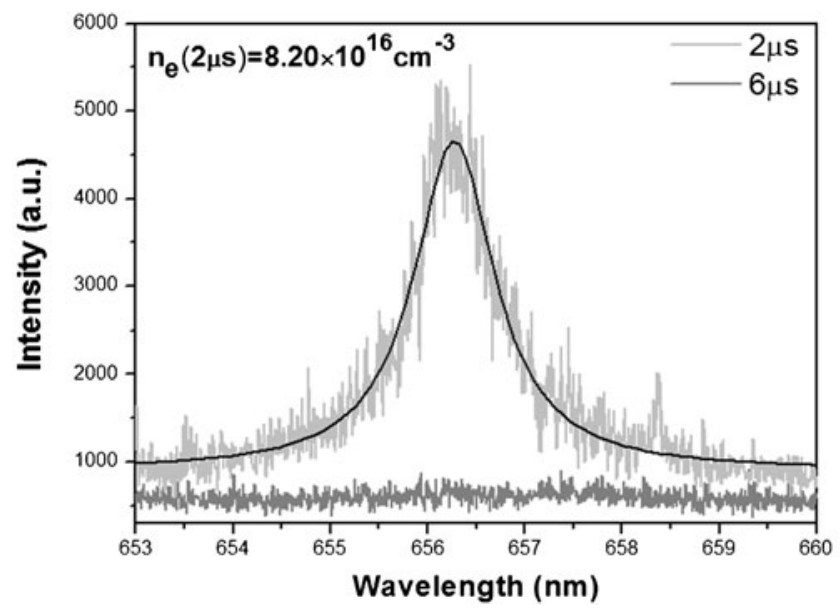

Fig. 8 Voigt fit to the observed experimental data for $\mathrm{H} \alpha$-line at $656.279 \mathrm{~nm}$

slopes of the lines, which determine the temperature, are distinct. The spectrum with $6 \mu$ s delay yield $T_{\mathrm{b}}=7,986 \mathrm{~K}$, lower as expected than the $2 \mu \mathrm{s}$ delay temperature $T_{\mathrm{a}}=12,490 \mathrm{~K}$. We will use these results from the wellestablished Saha-Boltzmann procedure to gauge the accuracy of our method.

To recapitulate, in Section 2, we have proposed a procedure to estimate the electron temperature of a time-dependent plasma. In this approach, to determine the plasma temperature, we again record spectra at two different time delays, with an spectrometer that needs not be calibrated. In the experimental study, we have adjusted the Voigt profile to measure the cadmium-emission line intensities. From the intensities we have obtained the parameters $q_{1}, q_{2}$, and $m$, which were substituted on the right-hand sides of Eqs. (7) and (9) to estimate the temperatures $T_{\mathrm{a}}$ and $T_{\mathrm{b}}$.

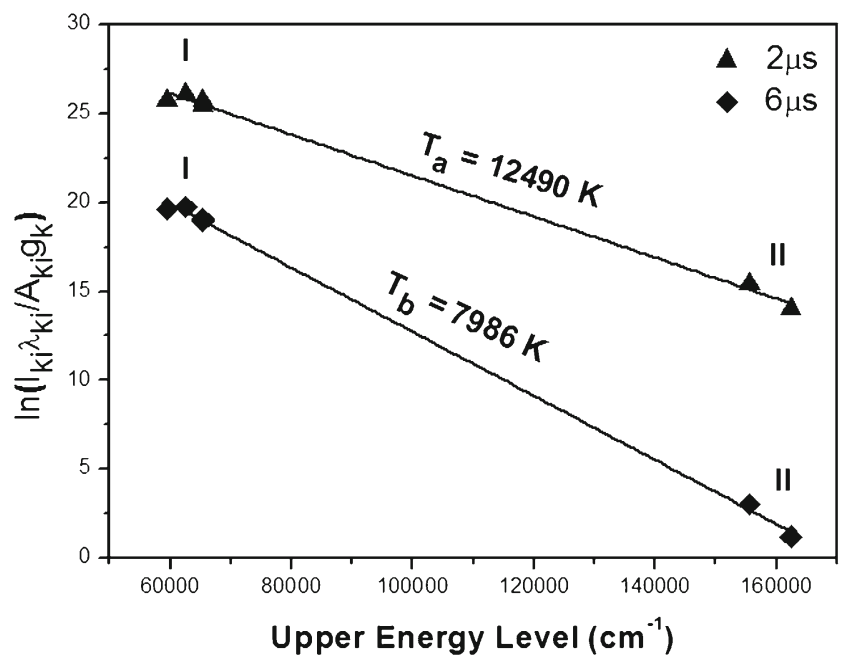

Fig. 9 Saha-Boltzmann plot obtained for two different delay times. The temperature value deduced from each plot is indicated

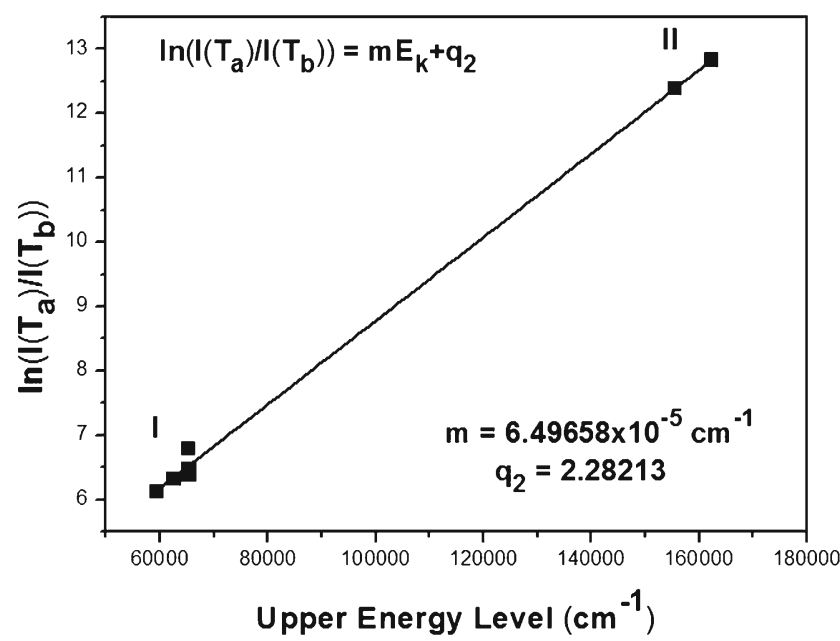

Fig. 10 Saha-Boltzmann plot obtained from the intensities ratio between the atomic/ionic cadmium lines at two different temperatures: $T_{\mathrm{a}}(2 \mu \mathrm{s})$ and $T_{\mathrm{b}}(6 \mu \mathrm{s})$

This procedure is described by Fig. 10, which shows the Saha-Boltzmann plot for the ratio of the spectral intensities with 2 and $6 \mu$ s time delay, from which the slope $m=6.49658 \times 10^{-5} \mathrm{~cm}^{-1}$ and interception point $q_{2}=2.28213$ are obtained. The large separation between the filled squares in the figure affords very accurate results for $\mathrm{m}$ and $\mathrm{q}_{2}$.

The intersection $q_{1}=6.99358$ is obtained from a plot Boltzmann of the ratio between the $\mathrm{Cd}$ II intensities. Figure 11 shows the Boltzmann plot for the emission-lines ratio for neutral and ionized cadmium. Straight lines with slope $m=6.49658 \times 10^{-5} \mathrm{~cm}^{-1}$ were drawn through the filled triangles and diamonds.

To estimate the two temperatures $T_{\mathrm{a}}(2 \mu \mathrm{s})$ and $T_{\mathrm{b}}(6 \mu \mathrm{s})$, Eq. (9) calls for the density ratio $n_{\mathrm{e}}\left(T_{\mathrm{a}}\right) / n_{\mathrm{e}}\left(T_{\mathrm{b}}\right)$. Our method is very sensitive to errors in this quotient. As an illustration, we have estimated $n_{\mathrm{e}}\left(T_{\mathrm{a}}\right) / n_{\mathrm{e}}\left(T_{\mathrm{b}}\right)$ in different ways. The first

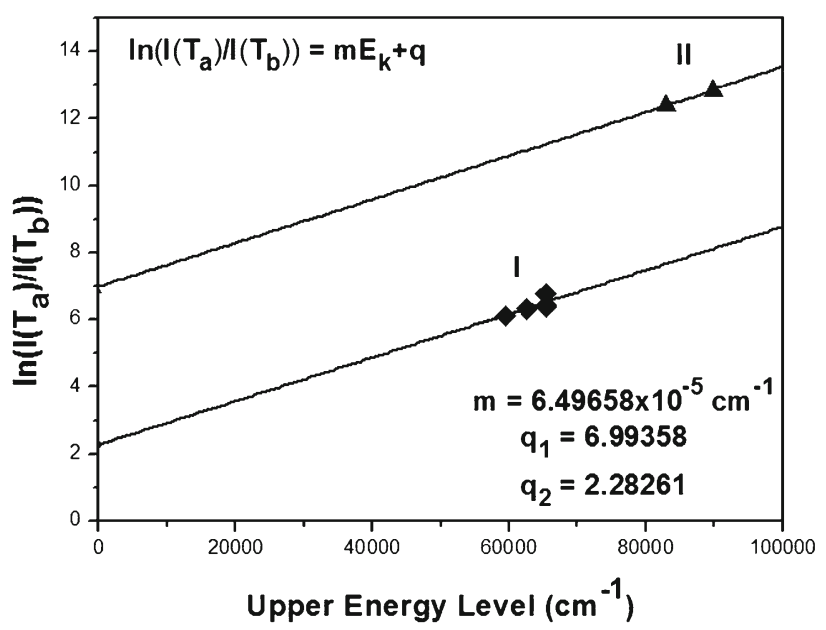

Fig. 11 Boltzmann plot containing data resulting from the intensities ratio for the $\mathrm{Cd} \mathrm{I}$ and $\mathrm{Cd}$ II. The slope was fixed at $6.49658 \times 10^{-5} \mathrm{~cm}^{-1}$ 


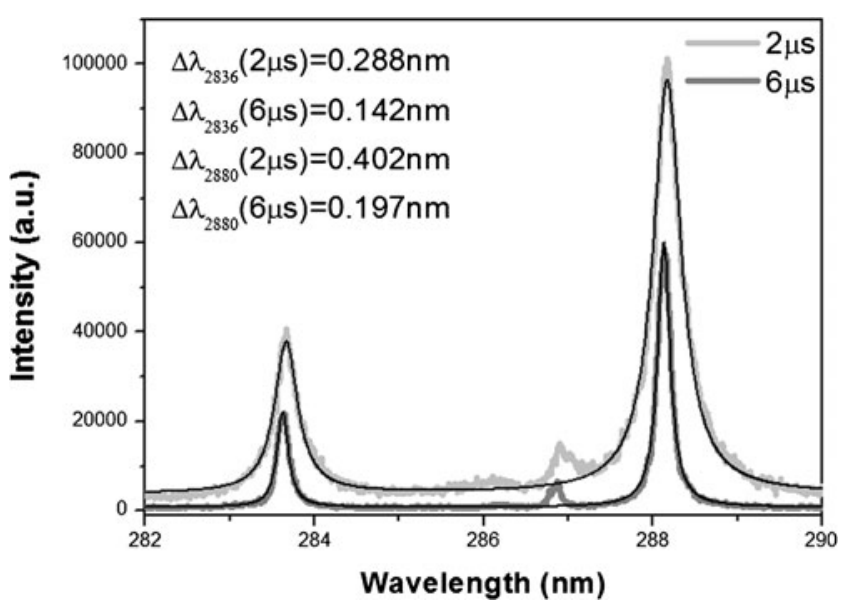

Fig. 12 Experimental profile of the Cd I lines at 283.690 and $288.076 \mathrm{~nm}$ in different temperatures. Both profile are fitted to Voigt profiles

estimate was obtained from the electron densities determined by the $\mathrm{H} \alpha$-line broadening in the $2 \mu$ s experiment and the electron density calculated by the Saha equation in the $6 \mu$ s measurement. From these values, we obtain $n_{\mathrm{e}}\left(T_{\mathrm{a}}\right) /<n_{\mathrm{e}}\left(T_{\mathrm{b}}\right)>=2.09$, a ratio that yields $T_{\mathrm{a}}(2 \mu \mathrm{s})=$ $14,028 \mathrm{~K}$ and $T_{\mathrm{b}}(6 \mu \mathrm{s})=8,588 \mathrm{~K}$, which are 12.3 and $7.5 \%$ off the temperatures obtained from the SahaBoltzmann plot, respectively.

A second estimate, $<n_{\mathrm{e}}\left(T_{\mathrm{a}}\right)>/<n_{\mathrm{e}}\left(T_{\mathrm{b}}\right)>=2.23$, was calculated from the densities given by the Saha equation. This ratio yields $T_{\mathrm{a}}=15,625 \mathrm{~K}$ and $T_{\mathrm{b}}=9,161 \mathrm{~K}$, with 25.1 and $14.7 \%$ deviations, respectively, from the temperatures obtained from the conventional method. For typical plasma LIBS, as in this application, the contribution from ion broadening is typically negligible. The contribution from collisions is almost entirely due to electron impact, and the half-width of the Stark-broadened transition can be estimated from the following linear approximation [12]:

$n_{\mathrm{e}} \approx \frac{\Delta \lambda_{\text {Stark }} \times 10^{16}}{2 w}$

were $\mathrm{n}_{\mathrm{e}}$ is the electron density in $\mathrm{cm}^{-3}$, and $\mathrm{w}$ is the electron impact parameter, a slowly varying function of the electron temperature that is independent of the electron density. From Eq. (12), we have the approximate equality $n_{e}\left(T_{a}\right) / n_{e}\left(T_{b}\right) \approx \Delta \lambda\left(T_{a}\right) / \Delta \lambda\left(T_{b}\right)$. The Stark profiles of a spectral line at the two temperatures $T_{\mathrm{a}}$ and $T_{\mathrm{b}}$ are therefore sufficient to yield an estimate of the ratio between electron densities.

Since the linewidth is affected by self-absorption, to determine the Stark-width ratio we have to seek a nonselfabsorbed emission line. The intensity ratio between lines connecting levels with similar upper-energy levels, i.e., lines belonging to the same multiplet, should be independent of the plasma temperature. Under experimental conditions, if the plasma temperature is the only time-dependent parameter, we expect the intensity ratio between such lines to be independent of the acquisition time delay. Among the lines in Table 2, we find no two lines belonging to the same multiplet. Nonetheless, the $2,836 \AA$ and $2,880 \AA$ lines have very close upper-energy levels, $65,353.372$ and $65,358.881 \mathrm{~cm}^{-1}$, and are good candidates for our test of self-absorption. The intensity ratio between these lines at different times are $I(2,880 \AA) / I(2,836 \AA)=3.01$ for $2 \mu \mathrm{s}$ and $I(2,880 \AA) / I(2,836 \AA)=3.00$ for $6 \mu \mathrm{s}$. We therefore chose calculated the Stark broadening for these lines.

Accurate measurement of the Stark broadening if the linewidth largely exceeds the instrumental broadening. Should this condition not be satisfied, unreliable values will result.

The experimental Stark broadenings, fitted to a Voigt profile, are shown in Fig. 12. The ratio between Stark broadenings for the $2,836 \AA$ line at the two temperatures is $\Delta \lambda_{2836}\left(T_{a}\right) / \Delta \lambda_{2836}\left(T_{b}\right)=2.03$, from which we find that $T_{\mathrm{a}}=13,332 \mathrm{~K}$ and $T_{\mathrm{b}}=8,322 \mathrm{~K}$, with deviations of 6.7 and $4.2 \%$ relative to the Saha-Boltzman plot values.

For the $2,880 \AA$ line, the calculated ratio is $\Delta \lambda_{2880}\left(T_{a}\right) / \Delta \lambda_{2880}\left(T_{b}\right)=2.04$, which corresponds and showed the following values; to $T_{\mathrm{a}}=13,448 \mathrm{~K}$ and $T_{\mathrm{b}}=8,367 \mathrm{~K}$, with relative errors of $7.7 \%$ and $4.8 \%$, respectively.

\section{Conclusions}

We have presented a procedure to estimate the temperature of a plasma that calls for no information on the spectral parameters - $A_{\mathrm{ki}}$, in particular - of the reference lines or on the calibration of the spectral detector. On the down side, the accuracy of the method is very sensitive to deviations in the electron number density and in the slope m. Equation (4) shows that the $\mathrm{m}$ deviations are strongly affected by uncertainties in the line intensities. To reduce those uncertainties, it is recommended that more than two lines be used to determine the slope $\mathrm{m}$ and that the resulting dispersion be analyzed.

Acknowledgment This work was supported by the National Autonomous University of Mexico PAPIIT \#IN114009. The authors also want to thank the Comisión de Investigaciones Científicas de la Provincia de Buenos Aires, where one of the authors, F. Bredice, works as a researcher. F.O. Borges thanks the Conselho Nacional de Desenvolvimento Científico e Tecnológico (CNPq) for a fellowship.

\section{References}

1. A. Miziolek, V. Palleschi, I. Schechter Edts, Laser-induced breakdown spectroscopy (LIBS) fundamentals and applications, chapter 3 (Cambridge University Press, Cambridge, 2006), pp. 129-136 
2. F. Bredice, H.O. Di Rocco, H.M. Sobral, M. Villagran-Muniz, V. Palleschi, Appl. Spectrosc. 64, 320-323 (2010)

3. D. M. D. Pace, G. Bertuccelli, and C. A. D'Angelo, Journal of Physics: Conference Series. 274, Article number 012076 (2011)

4. A. Ciucci, M. Corsi, V. Palleschi, S. Rastelli, A. Salvetti, E. Tognoni, Appl. Spectrosc. 53, 960-964 (1999)

5. B. Praher, V. Palleschi, R. Viskup, J. Heitz, J.D. Pedarnig, Spectrochimica Acta-Part B 65, 671-679 (2010)

6. E. Tognoni, G. Cristoforetti, S. Legnaioli, V. Palleschi, Spectrochimica Acta-Part B 65, 1-14 (2010)

7. G. Cristoforetti, A. De Giacomo, M. Dell'Aglio, S. Legnaioli, E. Tognoni, V. Palleschi, N. Omenetto, Spectrochimica Acta-Part B. 65, 86-95 (2010)

8. C. Aragón, J.A. Aguilera, Spectrochimica Acta-Part B. 63, 893$916(2008)$

9. S. Joshi, H. El-Rabii, C. Dumitrescu, P. Puzinauskas, A.P. Yalin, Spectrosc. Lett. 44, 103-112 (2011)

10. V.K. Unnikrishnan, K. Alti, V.B. Kartha, C. Santhosh, G.P. Gupta, B.M. Suri, Pramana. J. Phys. 74, 983-993 (2010)

11. G. Cristoforetti, S. Legnaioli, V. Palleschi, A. Salvetti, E. Tognoni, Spectrochimica Acta-Part B 63, 312-323 (2008)

12. F. Bredice, F.O. Borges, H. Sobral, M. Villagran-Muniz, H.O. Di Rocco, G. Cristoforetti, S. Legnaioli, V. Palleschi, L. Pardini, A. Salvetti, E. Tognoni, Spectrochimica Acta-Part B 61, 1294-1303 (2006)

13. H.E. Bauer, F. Leis, K. Niemax, Spectrochimica Acta-Part B 53, 1815-1825 (1998)

14. F. Bredice, F.O. Borges, H. Sobral, M. Villagran-Muniz, H.O. Di Rocco, G. Cristoforetti, S. Legnaioli, V. Palleschi, A. Salvetti, E. Tognoni, Spectrochimica Acta-Part B 62, 1237-1245 (2007)
15. E. Tognoni, V. Palleschi, M. Corsi, G. Cristoforetti, Spectrochimica Acta-Part B 57, 1115-1130 (2002)

16. S. Yalcin, D.R. Crosley, G.P. Smith, G.W. Faris, Appl. Phys. B. 68, 121-130 (1999)

17. J.A. Aguilera, C. Aragón, Spectrochimica Acta-Part B. 59, 18611876 (2004)

18. E. Kelleher, L.I. Podobedova, J. Phys, J. Phys. Chem. Ref. Data. 37, 709-911 (2008)

19. H. Di Rocco, F. Bredice, V. Palleschi, Appl. Spectrosc. 65, $1237-$ $1245(2011)$

20. J.A. Aguilera, C. Aragón, Spectrochimica Acta-Part B 62, 378 385 (2007)

21. A. El Sherbini, T. Sherbini, H. Hegazy, G. Cristoforetti, S. Legnaioli, L. Pardini, V. Palleschi, A. Salvetti, E. Tognoni, Spectrosc. Lett. 40, 643-658 (2007)

22. T. Anderson, G. Sorensen, J. Quant, Spectrosc. Radiat. Transfer 13, 369-376 (1973)

23. G. H. Corliss and W. R. Bozman, NBS Monograph 53 (Washington, DC: US Govet Pritning Office) (1962)

24. B. Warner, MNRAS 140, 53-59 (1968)

25. J. R. Fuhr, W. L. Wiese, in CRC Handbook of Chemistry and Physics, 86th Edition, 10-93-10-155 (Edited by D. R. Lide) CRC Press, Boca Raton; FL, 2005

26. A.M. El Sherbini, H. Hegaz, M. El Sherbini, Spectrochimica Acta-Part B 61, 532-539 (2006)

27. V.K. Unnikrishnan, V. Alti Kamlesh, B. Kartha, C. Santhosh, G.P. Gupta, B.M. Suri, Pramana. J. Phys. 74, 983-993 (2010)

28. J.A. Aguilera, C. Aragón, Spectrochimica Acta-Part B 62, 378385 (2007) 